PROCEEDINGS OF THE

AMERICAN MATHEMATICAL SOCIETY

Volume 128, Number 9, Pages 2789-2793

S 0002-9939(00)05310-7

Article electronically published on March 1, 2000

\title{
ON NON-ORIENTABLE SURFACES IN 4-SPACE WHICH ARE PROJECTED WITH AT MOST ONE TRIPLE POINT
}

\author{
SHIN SATOH
}

(Communicated by Ronald A. Fintushel)

\begin{abstract}
We show that if a non-orientable surface embedded in 4-space has a projection into 3-space with at most one triple point, then it is ambient isotopic to a connected sum of some unknotted projective planes and an embedded surface in 4-space with vanishing normal Euler number.
\end{abstract}

\section{INTRODUCTION}

In [4] Kinoshita constructed infinitely many projective planes embedded in $\mathbf{R}^{4}$ which are not ambient isotopic to each other. Each of his projective planes is a connected sum of an unknotted projective plane and a 2-knot. Price and Roseman gave a method to construct a projective plane in $\mathbf{R}^{4}$ from an invertible 1-knot and proved that every spun projective plane is ambient isotopic to a connected sum of an unknotted projective plane and a 2-twist spun 2-knot [6]. It is unknown whether there is an example of a projective plane in $\mathbf{R}^{4}$ which is not a connected sum of an unknotted projective plane and a 2-knot.

On the other hand, Yoshikawa gave infinitely many Klein bottles in $\mathbf{R}^{4}$ each of which is not ambient isotopic to a connected sum of an unknotted projective plane and a (possibly knotted) projective plane [7].

In this paper we discuss the problem of when a non-orientable surface in $\mathbf{R}^{4}$ is ambient isotopic to a connected sum of unknotted projective planes and a surface in $\mathbf{R}^{4}$ from the viewpoint of broken surface diagrams due to Carter and Saito [2].

A surface in $\mathbf{R}^{4}$ means a closed and connected PL 2-manifold without boundary embedded in $\mathbf{R}^{4}$ locally flatly. We call a surface in $\mathbf{R}^{4}$ a 2 -knot if the embedded surface is homeomorphic to a 2 -sphere. Moreover we can assume that the image $p(F)$ under the projection $p: \mathbf{R}^{4} \longrightarrow \mathbf{R}^{3}$ is a generic surface; that is, the singular set $\Gamma(p(F))$ on $p(F)$ consists of (possibly empty) isolated branch points, double point curves, and isolated triple points. Then we prove the following theorem.

Theorem 1.1. Let $F$ be a non-orientable surface in $\mathbf{R}^{4}$ and $e(F)$ its normal Euler number. If $\Gamma(p(F))$ contains at most one triple point, then $F$ is ambient isotopic to a connected sum of $|e(F)| / 2$ unknotted projective planes and a surface $F^{\prime}$ in $\mathbf{R}^{4}$ with $e\left(F^{\prime}\right)=0$.

Received by the editors July 20, 1998 and, in revised form, October 7, 1998.

1991 Mathematics Subject Classification. Primary 57Q45.

Key words and phrases. Non-orientable surface, connected sum, projective plane, triple point, branch point, normal Euler number. 
We notice that for Yoshikawa's Klein bottle $K b, \Gamma(p(K b))$ contains no triple point and $e(K b)$ is equal to zero.

Let $\chi$ be the Euler characteristic of a non-orientable surface $F$ in $\mathbf{R}^{4}$. Then the normal Euler number $e(F)$ is equal to $2 \chi-4,2 \chi, \cdots,-2 \chi$ or $4-2 \chi$ (cf. [5]). Since the normal Euler number of every projective plane is \pm 2 , we have the following.

Corollary 1.2. Let $P$ be a projective plane in $\mathbf{R}^{4}$. If $\Gamma(p(P))$ contains at most one triple point, then $P$ is ambient isotopic to a connected sum of an unknotted projective plane and a 2-knot.

\section{Projection With NO TRIPLE POINT}

Let $F$ be a surface in $\mathbf{R}^{4}$ and $p(F)$ its generic projection, where $p: \mathbf{R}^{4} \longrightarrow \mathbf{R}^{3}$ is the projection map.

The regular neighborhood in $\mathbf{R}^{3}$ of a branch point on $p(F)$ is as depicted in Figure 1(A). We define signs of branch points in such a way that a branch point with the crossing information depicted in Figure 1(B) receives the sign +1 , and one with the crossing information in Figure 1C) receives the sign -1 . Then the sum of signs taken over all the branch points in $\Gamma(p(F))$ is equal to the normal Euler number $e(F)$ of $F$ (cf. [1]).

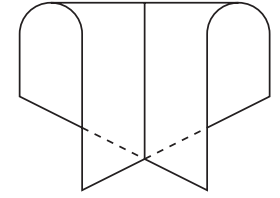

(A)

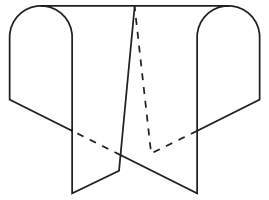

(B)

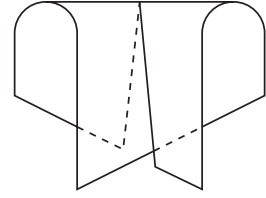

(C)

FIGURE 1.

A projective plane $P$ in $\mathbf{R}^{4}$ is unknotted if it is ambient isotopic to the surface whose projection into $\mathbf{R}^{3}$ is as depicted in Figure 2(A). There are two distinct unknotted projective planes, one of which has the normal Euler number $e(P)=+2$ and the other has $e(P)=-2$; see Figure 2(B) and (C). We denote these $P$ by $P_{+}$ (positive type) and $P_{-}$(negative type) respectively.

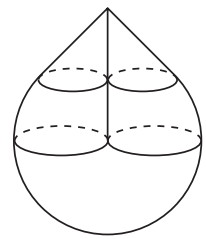

(A)

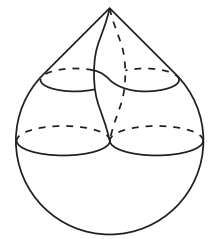

(B)

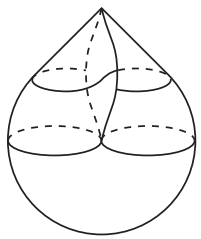

(C)

FIGURE 2.

Let $C_{1}, \cdots, C_{n}$ be the connected components of the singular set $\Gamma(p(F))$. Each $C_{i}$ is regarded as a union of immersed loops and immersed $\operatorname{arcs}$ in $\mathbf{R}^{3}$ such that the endpoints of the immersed arcs are branch points. Suppose that $C_{i}$ is one simple arc. Such a simple arc is called an $m$-arc (resp. $a$-arc) if the two branch points of 
its ends have the same sign (resp. opposite signs). Notice that the neighborhood of an $m$-arc (resp. $a$-arc) is homeomorphic to a Möbius band (resp. an annulus). Call an $m$-arc positive if it terminates with both positive type, or negative if it terminates with negative type. These terms are used in [3].

Lemma 2.1. Let $F$ be a non-orientable surface in $\mathbf{R}^{4}$. If $\Gamma(p(F))$ contains no triple point, then $F$ is ambient isotopic to a connected sum of $|e(F)| / 2$ unknotted projective planes and a surface $F^{\prime}$ in $\mathbf{R}^{4}$ with $e\left(F^{\prime}\right)=0$.

Proof. It is sufficient to prove the lemma in the case that $e(F)>0$. Since $\Gamma(P(F))$ contains no triple point, $\Gamma(P(F))$ is a disjoint union of simple loops, $a$-arcs and $m$-arcs. Notice that $e(F)=2(p-n)$, where $p$ is the number of positive $m$-arcs and $n$ is the number of negative $m$-arcs in $\Gamma(p(F))$. Then $\Gamma(p(F))$ contains at least $e(F) / 2$ positive $m$-arcs, and so $F$ is ambient isotopic to a connected sum of $e(F) / 2$ $P_{+}$and a surface $F^{\prime}$ in $\mathbf{R}^{4}$. Since $\Gamma\left(p\left(F^{\prime}\right)\right)$ is obtained from $\Gamma(p(F))$ by deleting the $e(F) / 2$ positive $m$-arcs, we have $e\left(F^{\prime}\right)=0$.

\section{Projection With ONE TRIPLE POINT}

Let $F$ be a surface in $\mathbf{R}^{4}$ and $p(F)$ its generic projection where $p: \mathbf{R}^{4} \longrightarrow \mathbf{R}^{3}$ is a projection map. The neighborhood of a triple point on $p(F)$ consists of three sheets. These sheets can be labeled top, middle and bottom, and these indicate the relative position of the sheets with respect to the projection direction.

The following is the key lemma in this paper.

Lemma 3.1. (1) Let $S$ be a generic closed surface in $\mathbf{R}^{3}$ and $c$ a simple closed curve in $\mathbf{R}^{3}$ such that $c$ is transverse to $S$ with $c \cap \Gamma(S)=\varnothing$. Then the number of $c \cap S$ is even.

(2) Let $t$ be a triple point in $\Gamma(p(F))$ and $H$ one of the three sheets around $t$. Suppose that there exists a subarc $\alpha:[0,1] \longrightarrow \Gamma(p(F))$ such that $\alpha(0)=\alpha(1)=t$, such that $\alpha([0, \varepsilon]) \subset H, \alpha([1-\varepsilon, 1]) \subset H$ for a small $\varepsilon>0$, and such that $\alpha($ int $[0,1])$ contains no triple point. Then $H$ is a top sheet or a bottom sheet.

Proof. (1) Since the $\mathbf{Z}_{2}$-intersection number of $c$ and $S$ in $\mathbf{R}^{3}$ is zero, the number of $c \cap S$ is even.

(2) There are two cases as depicted in Figure 3, where the shaded sheet represents the sheet $H$. Suppose that $H$ is a middle sheet in each case. Then its broken surface diagram is as depicted in Figure 4. Therefore the pasting map in the figure must be a rotation by $0^{\circ}$ or $180^{\circ}$ in Figure 4(A) and $\pm 90^{\circ}$ in Figure 4(B). This contradicts (1).

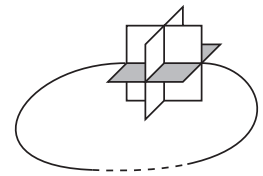

(A)

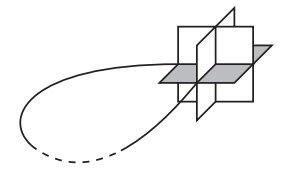

(B)

FiguRE 3. 


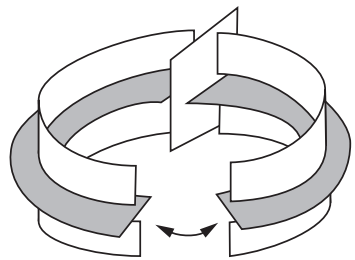

(A)

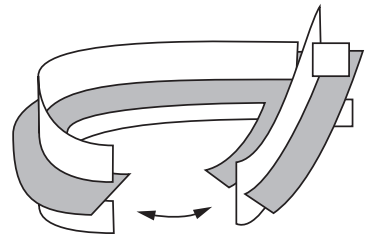

(B)

FIGURE 4.

We notice that in Lemma 3.1 2), even if $H$ is a top sheet or a bottom sheet, the case in Figure 4(A) does not occur.

The following lemma is proved by performing a sequence of two Roseman moves - "a sheet moving through a saddle point" and "passing a branch point through a third sheet", where the Roseman moves are generalizations of the Reidemeister moves and are sufficient to move embedded surfaces in $\mathbf{R}^{4}$. The list of the Roseman moves appears in [2].

Lemma 3.2. Let $t$ be a triple point in $\Gamma(p(F))$ and $H_{i}(i=1,2,3)$ three sheets around t. Suppose that the small arc $\mathrm{H}_{2} \cap \mathrm{H}_{3}$ is extended to an immersed arc $\gamma$ in $\Gamma(p(F))$ which ends in two branch points $b$ and $b^{\prime}$ and that the subarc $\gamma^{\prime}$ of $\gamma$ connecting between $t$ and $b$ contains no triple point inside. If $H_{1}$ is a top sheet or a bottom sheet, then the triple point $t$ can be removed from $p(F)$ by deforming $F$ by an ambient isotopy of $\mathbf{R}^{4}$ without introducing new triple points.

Proof. Let $D$ be a small neighborhood of $t$ in $H_{1}$. Push $D$ along $\gamma^{\prime}$ towards $b$. Since $H_{1}$ is a top sheet or a bottom sheet, we can push out $D$ over $b$. This deformation can be realized by an ambient isotopy of $\mathbf{R}^{4}$ and does not introduce new triple points in the projection. See Figure 5 (the figure is in the case that $H_{1}$ is a top sheet).

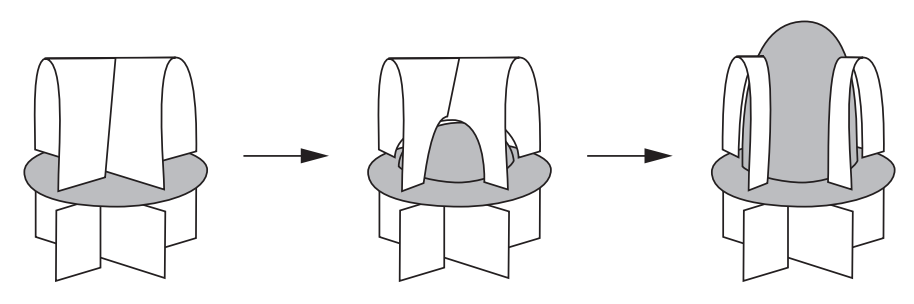

FiguRe 5.

Proof of Theorem [1.1. By Lemma 2.1, it is sufficient to consider the case that $\Gamma(p(F))$ contains just one triple point $t$. Let $C_{1}, \cdots, C_{n}$ be the connected components of $\Gamma(p(F))$ with $t \in C_{1}$.

If $C_{1}$ is a union of immersed loops, then all the branch points in $\Gamma(p(F))$ belong to $C_{2} \cup \cdots \cup C_{n}$, where each of $C_{2}, \cdots, C_{n}$ is a simple loop or a simple arc. Then we can apply an argument similar to the argument in the proof of Lemma 2.1$]$

Suppose that $C_{1}$ contains at least one immersed arc, say $\gamma$. We will prove that $t$ can be removed from $C_{1}$ without introducing new triple points. Let $c:[0,1] \longrightarrow \mathbf{R}^{3}$ 


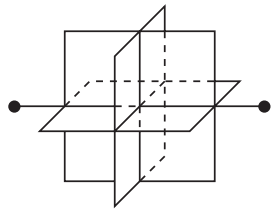

(A)

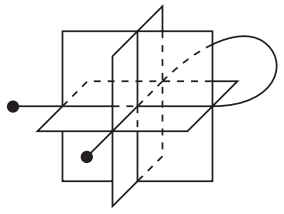

(B)

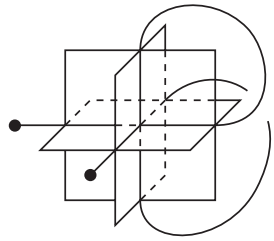

(C)

FiguRE 6.

be an immersion representing $\gamma$. First consider the case that $c^{-1}(t)$ consists of two or three points; see Figure6(B) and (C). Then we can perform the deformation in Lemma 3.2 so that $t$ can be removed from $C_{1}$. Consider the case that $c^{-1}(t)$ is one; see Figure 6(A). If the perpendicular sheet to $\gamma$ is top or bottom, then we can also perform the deformation in Lemma 3.2. If the perpendicular sheet is middle, then $C_{1}$ has the only form which consists of three arcs intersecting at $t$ by Lemma 3.1 Therefore we can perform the deformation in Lemma 3.2 .

\section{REFERENCES}

[1] Carter, J. Scott, and Saito, Masahico, Canceling branch points on projections of surfaces in 4-space, Proc. of the AMS. 116, No 1 (Sept 1992), pp. 229-237. MR 93i:57029

[2] — Reidemeister moves for surface isotopies and their interpretation as moves to movies, J. of Knot Theory and its Ramifications 2 (1993), pp. 251-284. MR 94i:57007

[3] - Normal Euler classes of knotted surfaces and triple points on projections, Proc. of the AMS. 125, No 2 (Feb 1997), pp. 617-623. MR 97d:57030

[4] S. Kinoshita, On the Alexander polynomials of 2-spheres in a 4-sphere, Ann. of Math. 74, No 3 (Nov 1961), pp. 518-531. MR 24:A2960

[5] W. S. Massey, Proof of a conjecture of Whitney, Pacific J. Math. 31 (1969), pp. 143-156. MR 40:3570

[6] T. M. Price and D. Roseman, Embeddings of the projective plane in four space, preprint.

[7] K. Yoshikawa, The order of a meridian of a knotted Klein bottle, preprint.

Department of Mathematics, Osaka City University, Sugimoto, Sumiyoshi-Ku, Osaka, 558-5858, JAPAN

E-mail address: susato@sci.osaka-cu.ac.jp 